\title{
Impact of the Lockdown Due to the COVID-19 Pandemic on Patients With Inflammatory Bowel Disease
}

\author{
Yu Nishida ${ }^{1}$, Shuhei Hosomi ${ }^{1 *}$, Koji Fujimoto ${ }^{1}$, Rieko Nakata ${ }^{1}$, Naoko Sugita ${ }^{1}$, \\ Shigehiro Itani ${ }^{1}$, Yuji Nadatani ${ }^{1}$, Shusei Fukunaga ${ }^{1}$, Koji Otani ${ }^{1}$, Fumio Tanaka ${ }^{1}$, \\ Yasuaki Nagami ${ }^{1}$, Koichi Taira ${ }^{1}$, Noriko Kamata ${ }^{1}$, Toshio Watanabe ${ }^{1}$, Satoko Ohfuji ${ }^{2}$ and \\ Yasuhiro Fujiwara ${ }^{1}$ \\ ${ }^{1}$ Department of Gastroenterology, Osaka City University Graduate School of Medicine, Osaka, Japan, ${ }^{2}$ Department of Public \\ Health, Osaka City University Graduate School of Medicine, Osaka, Japan
}

Background: The government of Japan declared a state of emergency on April 16, 2020, owing to the coronavirus disease 2019 (COVID-19) pandemic. The subsequent lockdown altered lifestyles and worsened mental illnesses. Inflammatory bowel disease (IBD) is an intestinal disorder that is affected by environmental factors. Therefore, we aimed to assess the effects of COVID-19 and the state of emergency on the lifestyle and disease activity of patients with IBD.

Methods: We conducted a questionnaire survey on patients with IBD from June 16 to August 21, 2020 during their regular follow-up at our hospital, 2 months after the state of emergency was declared.

Results: Overall, 241 patients with ulcerative colitis (UC) and 210 with Crohn's disease (CD) completed the survey, of which 82 (34\%) and 97 (46\%) patients, respectively, reported disease exacerbation within 2 months after the lockdown. Multivariate logistic regression analysis identified age at enrollment (odds ratio, OR $0.98,95 \% \mathrm{Cl} 0.96-$ 0.99; $P<0.05$ ), sleep hours (OR, 0.74; 95\% Cl, 0.57-0.97; $P<0.05)$, and increased stress due to the COVID-19 pandemic (OR, 6.06; 95\% Cl, 1.79-20.50; $P<0.01)$ as independent factors associated with UC exacerbation. Patients with exacerbated CD were younger at $C D$ onset and had higher patient-reported outcome 2 scores before the state of emergency than patients with non-exacerbated CD. On multivariate analysis, age (OR, 0.97; 95\% Cl, 0.95-0.99; $P<0.01)$ and active disease before the state of emergency (OR, 2.20; 95\% Cl, 1.23-3.95; $P<0.01$ ) were independently associated with CD exacerbation.

Conclusions: Improving sleep quality and preventing psychological stress may be crucial in IBD management during a pandemic, especially in young patients.

Keywords: COVID-19, inflammatory bowel disease, lockdown, ulcerative colitis, Crohn's disease

\section{INTRODUCTION}

In December 2019, coronavirus disease 2019 (COVID-19), which is caused by severe acute respiratory syndrome coronavirus 2 (SARS-CoV-2), emerged in Wuhan, China, and the outbreak rapidly spread worldwide (1). It was considered a global health emergency by the World Health Organization. One measure that was adopted by the governments of many countries, especially 
those more affected by the pandemic, was the lockdown of cities. Consistent with other countries' policies, the government of Japan declared a state of emergency on April 16, 2020, which continued until May 25, 2020. Central and local governments could request or instruct behaviors to prevent the spread of infection, such as school closure, social distancing, and quarantine. Although this approach was partially successful in temporarily preventing the spread (2), concerns were raised regarding the negative impact of these measures not only in terms of economics but also for mental and physical health $(3,4)$. The lockdown altered sleep, exercise, and nutrition patterns; compromised treatment compliance; increased childcare and work burden (owing to the lack of a workforce); and worsened mental illnesses, such as anxiety and depression (5-8).

Inflammatory bowel diseases (IBDs), comprising ulcerative colitis (UC) and Crohn's disease (CD), are intestinal disorders affected by environmental factors, such as sleep, stress, diet, and smoking (9-13). However, few studies have evaluated the relationship between lockdown measures to control the COVID19 pandemic and IBD exacerbation. Therefore, this study aimed to assess the effects of the COVID-19 pandemic and state of emergency on the lifestyle and disease activity of patients with IBD.

\section{MATERIALS AND METHODS \\ Study Design and Participants}

This study was conducted through a questionnaire survey among patients with IBD during their regular follow-up at a hospital in Japan, 2 months after the initiation of the state of emergency (from June 16 to August 21, 2020). We asked all patients with IBD who visited the hospital during this period to complete the questionnaire. Patients with repeated visits were investigated only once. The exclusion criteria were a diagnosis of IBD in the last 3 months, inability to complete the questionnaire despite assistance, presence of colostomy or ileostomy, and history of total proctocolectomy with ileal pouch-anal anastomosis.

\section{Questionnaire Design}

The questionnaire included questions regarding the patient's epidemiological history of COVID-19, demographic data (sex, age at recruitment, and age at disease diagnosis), gastrointestinal symptoms, lifestyle (sleeping time, working time, walking time, exercise time, and number of meals) before and after the declaration of the state of emergency, stress related to the state of emergency (due to childcare burden), COVID-19, family budget, inability to exercise, staying indoors, IBD, and worsening of diet and nutritional status), and current medication use.

\section{Evaluation}

Gastrointestinal symptoms were assessed before and after the state of emergency (from April 16 to May 15) using the 6point Mayo score $(14,15)$ and patient-reported outcome 2

Abbreviations: COVID-19, coronavirus disease; CD, Crohn's disease; IBD, inflammatory bowel disease; OR, odds ratio; PRO2, patient-reported outcome 2; SARS-CoV-2, severe acute respiratory syndrome coronavirus 2; UC, ulcerative colitis.
(PRO2) score (16) for UC and CD, respectively. Severe active UC, moderate active UC, mild active UC, and UC remission were defined as a 6-point Mayo score of $\geq 5,3-4,2$ and $0-1$, respectively (15). Severe active $\mathrm{CD}$, moderate active $\mathrm{CD}$, mild active $\mathrm{CD}$, and $\mathrm{CD}$ remission were defined as a $\mathrm{PRO} 2$ score of $\geq 34,14-33,8-13$, and $0-7$, respectively. Patients with mild, moderate, or severe UC were defined as having active disease (16). The primary endpoint was disease exacerbation defined as an increase in the 6-point Mayo or PRO2 scores. "Stress related to the state of emergency" was defined as newly emerging stress during the state of emergency. Deterioration of adherence was defined as an increase in the number of times a patient forgot to take a prescribed medication within a week after the state of emergency.

\section{Statistical Analysis}

Continuous variables are summarized as medians and interquartile ranges. The differences in clinical characteristics were compared using either the chi-square test or Fisher's exact test for categorical variables and the Mann-Whitney $U$ test for continuous variables. Multivariate logistic regression analyses were performed to identify factors associated with exacerbation. Variables in the multivariate analysis were selected among those showing significant differences in a comparison between exacerbated and non-exacerbated patients, and based on known risk factors for exacerbation.

A $P$-value of $<0.05$ was considered significant. All statistical analyses were performed using EZR software (Saitama Medical Center, Jichi Medical University), a graphical user interface for $\mathrm{R}$ (The $\mathrm{R}$ Foundation for Statistical Computing, version 2.13.0). More precisely, it is a modified version of $\mathrm{R}$ commander (version 1.6-3), which includes statistical functions frequently used in biostatistics.

\section{RESULTS}

\section{Patients}

A total of 511 questionnaires were returned, of which 60 were excluded owing to missing items. Overall, 451 patients completed the survey. Two participants had come into close contact with confirmed cases of COVID-19, and one of these had undergone isolation; however, no cases of COVID-19 were enrolled in the study.

\section{Disease-Related Variables}

Regarding specific diagnosis and disease activity before lockdown, 241 patients had UC (remission, 213 [88.4\%]; mild activity, 14 [5.8\%]; moderate activity, 11 [4.6\%]; severe activity, $3[1.2 \%]$ ) and 210 patients had CD (remission, 123 [58.6\%]; mild activity, 46 [21.9\%]; moderate activity, 39 [18.6\%]; severe activity, $2[1.0 \%])$. The median age at enrollment was 50 years (IQR 39-63) for both patients with UC and CD. The median age at diagnosis was 31 years (IQR 24-42) in patients with UC and 25 years (IQR 19-33) in patients with CD. The median disease duration was 13 years for both patients with UC (IQR 7-23) and CD (IQR 6-24). The detailed characteristics of the patients are shown in Table 1. 
TABLE 1 | Demographic data and disease-related variables of participants.

\begin{tabular}{|c|c|c|c|}
\hline & & Ulcerative colitis & Crohn's disease \\
\hline \multirow[t]{9}{*}{ Demographics } & Number of patients & 241 & 210 \\
\hline & Sex (male/female) & $129 / 112$ & $158 / 52$ \\
\hline & Age at enrollment (years), median (IQR) & $50(39-63)$ & $44(34-50)$ \\
\hline & Age at diagnosis (years), median (IQR) & $31(24-42)$ & $25(19-33)$ \\
\hline & Disease duration (years), median (IQR) & $13(7-23)$ & $13(6-24)$ \\
\hline & 6-point Mayo score before the declaration of the state of emergency & $0(0-1)$ & \\
\hline & 6-point Mayo score during the state of emergency & $0(0-1)$ & \\
\hline & PRO2 score before the declaration of the state of emergency & & $6(0-11)$ \\
\hline & PRO2 score during the state of emergency & & $9(4-15)$ \\
\hline \multirow[t]{8}{*}{ Lifestyle during the state of emergency } & Sleeping time (hours/day), mean (IQR) & $6(6-7)$ & $6(6-7)$ \\
\hline & Working time (hours/week), median (IQR) & $12(0-40)$ & $8(0-8.75)$ \\
\hline & Walking time (hours/day), median (IQR) & $1(0-1)$ & $1(0-1)$ \\
\hline & Exercise time (minutes/week), median (IQR) & $0(0-120)$ & $10(0-40)$ \\
\hline & Number of meals per day, median (IQR) & $3(3-3)$ & $3(2-3)$ \\
\hline & Increased smoking & $1(0.4 \%)$ & $14(6.7 \%)$ \\
\hline & Increased alcohol intake & $29(12.0 \%)$ & $23(11.0 \%)$ \\
\hline & Deterioration of drug-adherence & $3(1.2 \%)$ & $2(1.0 \%)$ \\
\hline \multirow[t]{7}{*}{ Stress related to the state of emergency ${ }^{\dagger}$} & Stress due to childcare burden & $2(0.8 \%)$ & $0(0 \%)$ \\
\hline & Stress due to COVID-19 & $14(5.8 \%)$ & 7 (3.3\%) \\
\hline & Stress due to family budget & $10(4.1 \%)$ & $3(1.4 \%)$ \\
\hline & Stress due to inability to exercise & $21(8.7 \%)$ & $10(4.8 \%)$ \\
\hline & Stress due to staying indoors & $25(10.4 \%)$ & $18(8.6 \%)$ \\
\hline & Stress due to inflammatory bowel disease & $7(2.9 \%)$ & $3(1.4 \%)$ \\
\hline & Stress due to worsening of diet and nutritional status & $5(2.1 \%)$ & $2(1.0 \%)$ \\
\hline \multirow[t]{9}{*}{ Medication } & Mesalamine & $214(88.8 \%)$ & $123(58.6 \%)$ \\
\hline & Enteral nutrition & $0(0 \%)$ & $66(31.4 \%)$ \\
\hline & Corticosteroids & $8(3.3 \%)$ & $8(3.8 \%)$ \\
\hline & Immunomodulators (azathioprine or 6-mercaptopurine) & $64(26.6 \%)$ & $70(33.3 \%)$ \\
\hline & Anti-TNF therapy & $31(12.9 \%)$ & 109 (51.9\%) \\
\hline & Ustekinumab & $0(0 \%)$ & $26(12.4 \%)$ \\
\hline & Vedolizumab & $11(4.6 \%)$ & 7 (3.3\%) \\
\hline & Tofacitinib & $6(2.5 \%)$ & not approved in Japan* \\
\hline & Molecularly targeted therapies ${ }^{\star \star}$ & $48(19.9 \%)$ & $141(67.1 \%)$ \\
\hline
\end{tabular}

\footnotetext{
† "Stress related to the state of emergency" was defined as newly emerging stress during the state of emergency. *Tofacitinib is not approved for the treatment of Crohn's disease in Japan.

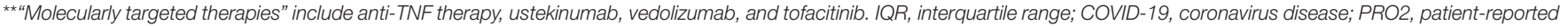
outcome 2; TNF, tumor necrosis factor.
}

\section{Impact of the Lockdown on Disease Activity, Lifestyle, and Psychological Stress}

Within 2 months after the declaration of the state of emergency, gastrointestinal symptoms worsened in $34.0 \%$ and $46.2 \%$ of patients with UC and $\mathrm{CD}$, respectively. Figure 1 shows a comparison of disease activity before and during lockdown. UC and $\mathrm{CD}$ activity after lockdown were as follows: UC (remission, 180 [74.7\%]; mild activity, 29 [12.0\%]; moderate activity, 22 [9.1\%]; severe activity, 10 [4.1\%]) and CD (remission, 96 [45.7\%]; mild activity, 53 [25.2\%]; moderate activity, 54 [25.7\%]; severe activity, $2[1.0 \%]$ ). Additional treatment was only required for $14.6 \%$ and $12.4 \%$ of patients with exacerbated UC and CD, respectively. Among 213 patients with UC and 123 patients with $\mathrm{CD}$ who were in remission before lockdown, gastrointestinal symptoms worsened in $71(33.3 \%)$ and 48 patients (39.0\%), respectively. The rate of disease exacerbation did not significantly differ between all participants and those in remission for UC $(P=0.921)$ and $\mathrm{CD}(P=0.21)$. In contrast, among 170 and 148 patients with UC and CD, respectively, who did not receive additional treatment due to disease exacerbation within 1 year before the state of emergency, gastrointestinal symptoms worsened in $54(31.8 \%)$ and 63 patients (42.6\%), respectively. The rate of disease exacerbation did not significantly differ between all participants and those with UC $(P=0.671)$ or $\mathrm{CD}$ $(P=0.519)$ who did not undergo additional treatment due to disease exacerbation within 1 year before the state of emergency. Regarding smoking, alcohol intake, and drug adherence, an increase in smoking was seen in $1(0.4 \%)$ and $14(6.7 \%)$, alcohol intake in $29(12.0 \%)$ and $23(11.0 \%)$, and a deterioration of drug-adherence in $3(1.2 \%)$ and $2(1.0 \%)$, UC and CD patients, 


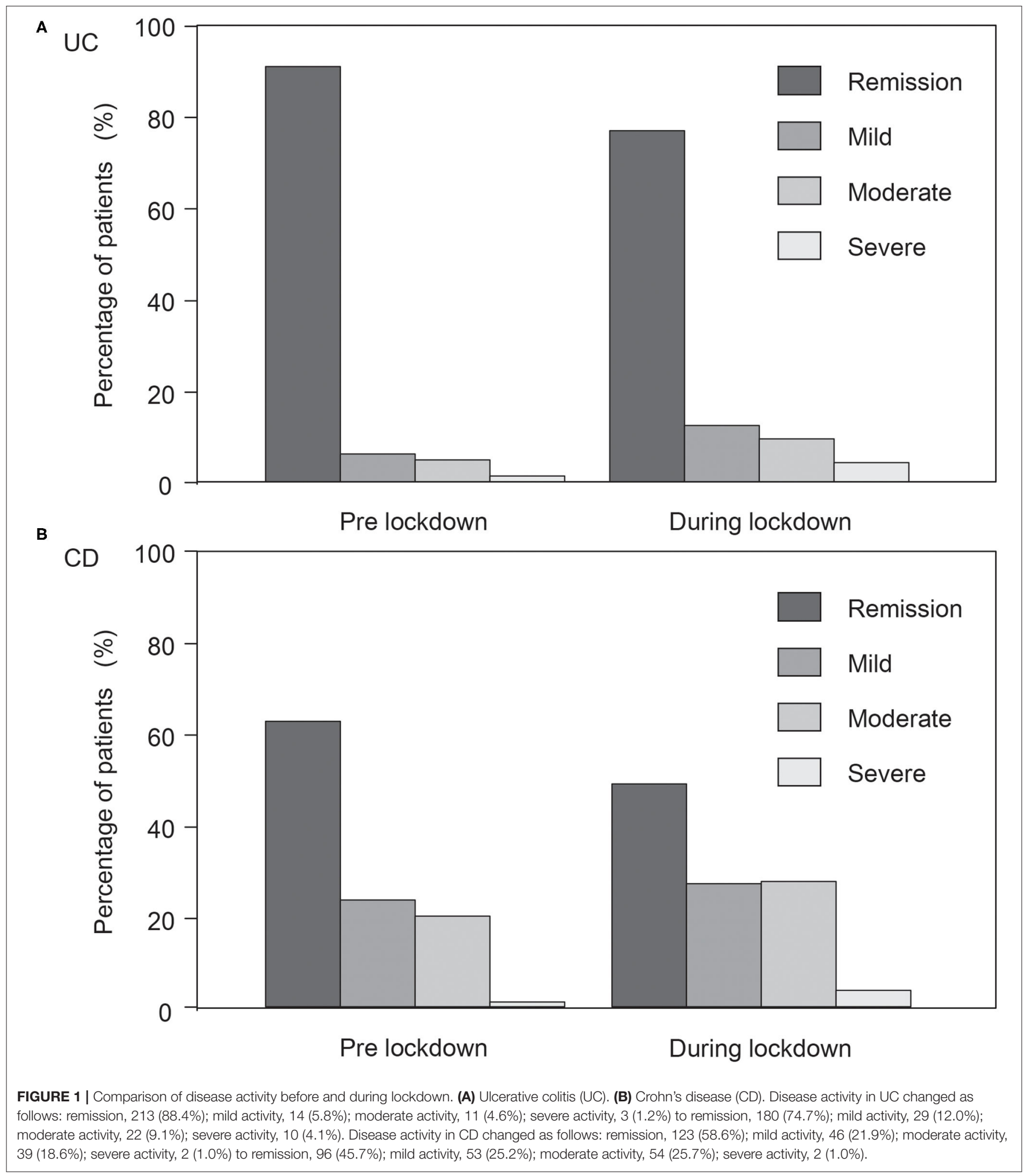

respectively. Regarding psychological stress, a high percentage of people felt stress due to being forced to stay indoors or the inability to exercise, whereas the proportion of people with stress due to IBD or nutrition was not significantly high (Table 1).

\section{Risk Factors for Exacerbation}

Table 2 shows a comparison of patient data. Patients with exacerbated UC (within 2 months after the declaration of the state of emergency) tended to be younger and had less 
sleep and more stress due to COVID-19 than patients with non-exacerbated UC. Multivariate logistic regression analysis identified age (odds ratio, OR 0.98, 95\% CI 0.96-0.99; $P<$ 0.05 ), sleep hours (OR, 0.74; 95\% CI, 0.57-0.97; $P<0.05$ ), and increased stress due to the COVID-19 pandemic (OR, 6.06; 95\% CI, 1.79-20.50; $P<0.01)$ as independent risk factors associated with UC exacerbation (Table 3). Regarding patients with CD, those with exacerbations were lower age at enrollment, lower age at $\mathrm{CD}$ onset, and had active disease before the state of emergency than patients with non-exacerbated CD. However, multivariate analysis identified age (OR, 0.97; 95\% CI, 0.95-0.99; $P<0.01)$ and active disease before the state of emergency (OR, 2.20; 95\% CI, 1.23-3.95; $P<0.01$ ) as independent factors associated with CD exacerbation (Table 3). Alcohol increase, smoking increase, and drug adherence change were not identified as independent risk factors for exacerbation.

\section{DISCUSSION}

Our results suggest that changes in daily life and stress status due to the pandemic and lockdown measures were associated with worsening IBD symptoms, especially in young patients. Possible explanations for these findings could be as follows. First, according to a recent report, the negative impact of lockdown measures on daily life may be more prevalent in younger people than in older people (4). The impact of age at IBD onset on the natural history, severity, and surgical rates have been reported to be higher in patients with elderly-onset UC than in patients with non-elderly-onset UC (17-20), whereas the rates of disease progression have been shown to be lower in patients with elderlyonset CD than in patients with non-elderly-onset $\operatorname{CD}(21,22)$. In the current study, although both patients with UC and CD with worsening IBD symptoms were younger than those without worsening symptoms at enrollment, only patients with CD with worsening symptoms were younger at $\mathrm{CD}$ onset than those without worsening symptoms. These results indicate that patients with UC might experience episodes of exacerbations due to the impact of the lockdown, but not natural history, in contrast to patients with CD.

Second, sleep disturbances are commonly seen in patients with active $\operatorname{IBD}(23,24)$ and are associated with the onset of UC (24). Ananthakrishnan et al. reported that sleep disturbance was associated with an increased risk of CD but not UC exacerbation (25). In the current study, multivariate logistic regression analysis identified fewer sleep hours as an independent risk factor associated with UC but not with CD exacerbation. This discrepancy may occur owing to the quality of sleep. Only sleep time could be evaluated as a sleep factor, as the questionnaire used in this study did not include questions associated with sleep disturbance or use of medications that could estimate the quality of sleep. Therefore, further studies will be required to explain this discrepancy.

Finally, stress resulting from the fear of contracting a potentially lethal disease that affects mostly immunosuppressed individuals might aggravate IBD symptoms, though an inverse relation cannot be excluded (IBD exacerbation could cause psychological stress). Several studies have reported the psychological impact of the pandemic on the general population and demonstrated an increase in the level of anxiety during the pandemic, and patients with IBD are more likely to develop anxiety disorders (26-28).

In this study, no case of COVID-19 was registered; however, this does not mean patients with IBD were less likely to contract COVID-19. This is probably owing to the small sample size and low infection rate of COVID-19 during this period in Japan. Indeed, IBD per se does not increase the risk of developing COVID-19 (29), and patients with IBD receiving immunomodulators, biological agents, or JAK inhibitors do not have an increased risk of contracting SARS-CoV-2 infection or developing a more severe course of infection (30). Only corticosteroid use was reported to be associated with severe COVID-19 among patients with IBD (31). However, elderly patients or those with comorbidities have a poorer clinical outcome after contracting COVID-19 (30, 32, 33). Based on this evidence and the results of our study, older patients or those with current use of corticosteroid treatments need thorough observation and early intervention to prevent the potential development of severe COVID-19. In addition, younger patients should be careful to prevent exacerbations of IBD during lockdown because they are likely to worsen. Additionally, the results of our study suggested that patients in remission or those who did not require additional treatment within 1 year before the state of emergency had a similar risk of disease exacerbation during the state of emergency. The multivariate logistic regression analysis that included scores for gastrointestinal symptoms also supported this finding.

This study has some limitations, including its single-center nature and relatively small cohort, which could be prone to bias in data selection and analysis. Moreover, the results of our study should have been compared with the rate and factors for gastrointestinal disease exacerbation before the COVID19 pandemic occurred; however, this comparison could not be performed because of the lack of relevant pre-COVID-19 pandemic data for these diseases. Additionally, we were unable to evaluate objective factors (e.g., laboratory examinations, endoscopic activities, disease locations) because anonymity needed to be maintained in the questionnaire. Patients who experienced disease exacerbation may have had a functional disorder, but it was difficult to assess the influence of such based only on subjective factors. Only 14.6 and $12.4 \%$ of patients with exacerbated UC and CD, respectively, required additional treatment, some of which may have had a functional disorder or experienced mild exacerbation of the disease. Disease exacerbation in this study was defined as an increase of 1 point or more in the 6-point Mayo or PRO2 scores. However, since disease exacerbation was not evaluated objectively, it could not be quantified. Further, change in the line of treatments for IBD flare-up during the COVID-19 pandemic should have been evaluated, as alterations could have been made throughout this period that may have affected our results. For example, the European Crohn's and Colitis Organization position statement recommended the use of subcutaneous drugs for IBD flare-ups to minimize hospital visits (34). However, we could not evaluate 
TABLE 2 | Descriptive comparison of participants with and without exacerbation.

\begin{tabular}{|c|c|c|c|c|c|c|c|}
\hline & & \multicolumn{3}{|c|}{ Patients with ulcerative colitis } & \multicolumn{3}{|c|}{ Patients with Crohn's disease } \\
\hline \multirow[t]{6}{*}{ Demographics } & Number of patients & 159 & 82 & & 113 & 97 & \\
\hline & Sex (male/female) & $82 / 77$ & $47 / 35$ & 0.416 & $85 / 28$ & $73 / 24$ & 1 \\
\hline & Age at enrolment (years), median (IQR) & $51(39-66)$ & $\begin{array}{c}46.5 \\
(39-56.5)\end{array}$ & 0.051 & $46(35-54)$ & $42(31-48)$ & 0.014 \\
\hline & Disease duration (years), median (IQR) & $13(7-23)$ & $\begin{array}{c}13.5 \\
(5-22.25)\end{array}$ & 0.533 & $13(5-25)$ & $15(7-22)$ & 0.788 \\
\hline & $\begin{array}{l}\text { 6-point Mayo score before the declaration } \\
\text { of the state of emergency }\end{array}$ & $0(0-1)$ & $0(0-1)$ & 0.221 & & & \\
\hline & $\begin{array}{l}\text { PRO2 score before the declaration of the } \\
\text { state of emergency }\end{array}$ & & & & $5(0-10)$ & $8(2-13)$ & 0.025 \\
\hline & Walking time (hours/day), median (IQR) & $1(0-1)$ & $1(0-1)$ & 0.295 & $1(1-1)$ & $1(0-1)$ & 0.491 \\
\hline & $\begin{array}{l}\text { Exercise time (minutes/week), median } \\
\text { (IQR) }\end{array}$ & $0(0-120)$ & $0(0-120)$ & 0.917 & $10(0-60)$ & $15(0-30)$ & 0.819 \\
\hline & Number of meals per day, median (IQR) & $3(3-3)$ & $3(3-3)$ & 0.493 & $3(2-3)$ & $3(2-3)$ & 0.593 \\
\hline & Increased smoking & $1(0.6 \%)$ & $0(0.0 \%)$ & 1 & $8(7.1 \%)$ & $6(6.2 \%)$ & 1 \\
\hline & Increased alcohol intake & $17(10.7 \%)$ & $12(14.6 \%)$ & 0.406 & $12(10.6 \%)$ & $11(11.3 \%)$ & 1 \\
\hline & Deterioration of drug-adherence & $1(0.6 \%)$ & $2(2.4 \%)$ & 0.268 & $1(1.0 \%)$ & $1(1.1 \%)$ & 1 \\
\hline \multirow{3}{*}{$\begin{array}{l}\text { Stress related to the } \\
\text { state of emergency }\end{array}$} & Stress due to childcare burden & $1(0.6 \%)$ & $1(1.2 \%)$ & 1 & $0(0 \%)$ & $0(0 \%)$ & NA \\
\hline & Stress due to COVID-19 & $4(2.5 \%)$ & $10(12.2 \%)$ & 0.006 & $2(1.8 \%)$ & $5(5.2 \%)$ & 0.253 \\
\hline & Stress due to family budget & $7(4.4 \%)$ & $3(3.7 \%)$ & 1 & $1(0.9 \%)$ & $2(2.1 \%)$ & 0.597 \\
\hline \multirow{7}{*}{ Medication } & Corticosteroids & $5(3.1 \%)$ & $3(3.7 \%)$ & 1 & $3(2.7 \%)$ & $5(5.2 \%)$ & 0.475 \\
\hline & $\begin{array}{l}\text { Immunomodulators (azathioprine or } \\
\text { 6-mercaptopurine) }\end{array}$ & 47 (29.6\%) & $17(20.7 \%)$ & 0.167 & $35(31 \%)$ & 35 (36.1\%) & 0.465 \\
\hline & Anti-TNF therapy & $17(10.7 \%)$ & $14(17.1 \%)$ & 0.222 & $55(48.7 \%)$ & $54(55.7 \%)$ & 0.335 \\
\hline & Ustekinumab & $0(0 \%)$ & $0(0 \%)$ & NA & $13(11.5 \%)$ & $13(13.4 \%)$ & 0.681 \\
\hline & Vedolizumab & $8(5 \%)$ & $3(3.7 \%)$ & 0.754 & $2(1.8 \%)$ & $5(5.2 \%)$ & 0.253 \\
\hline & Tofacitinib & $3(1.9 \%)$ & $3(3.7 \%)$ & 0.404 & \multicolumn{3}{|c|}{ Not approved in Japan } \\
\hline & Molecularly targeted therapies ${ }^{\star *}$ & $28(17.6 \%)$ & 20 (24.4\%) & 0.235 & 70 (61.9\%) & $71(73.2 \%)$ & 0.105 \\
\hline
\end{tabular}

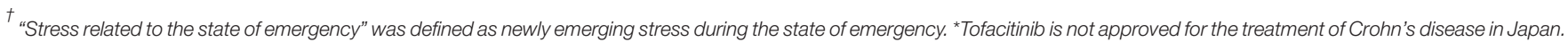
**"Molecularly targeted therapies" include anti-TNF therapy, ustekinumab, vedolizumab, and tofacitinib. COVID-19, coronavirus disease; IQR, interquartile range; PRO2, patient-reported outcome 2; TNF, tumor necrosis factor.
}

the details of the additional treatment for the exacerbation because the questionnaire did not include these items. Further, we used simple questions rather than validated ones for psychological factors to reduce the burden on respondents and increase the response rate in consideration of the large number of questions.
Another possible limitation of this study was possible selection bias. Since patients visiting the clinic are likely to have more symptoms (or less), the results may be biased and not generalizable to all patients with IBD. In addition, this study was conducted in a single-tertiary center, which may suggest the patients have more complicated disease. However, in Japan, 
TABLE 3 | Logistic regression analyses of factors associated with exacerbation.

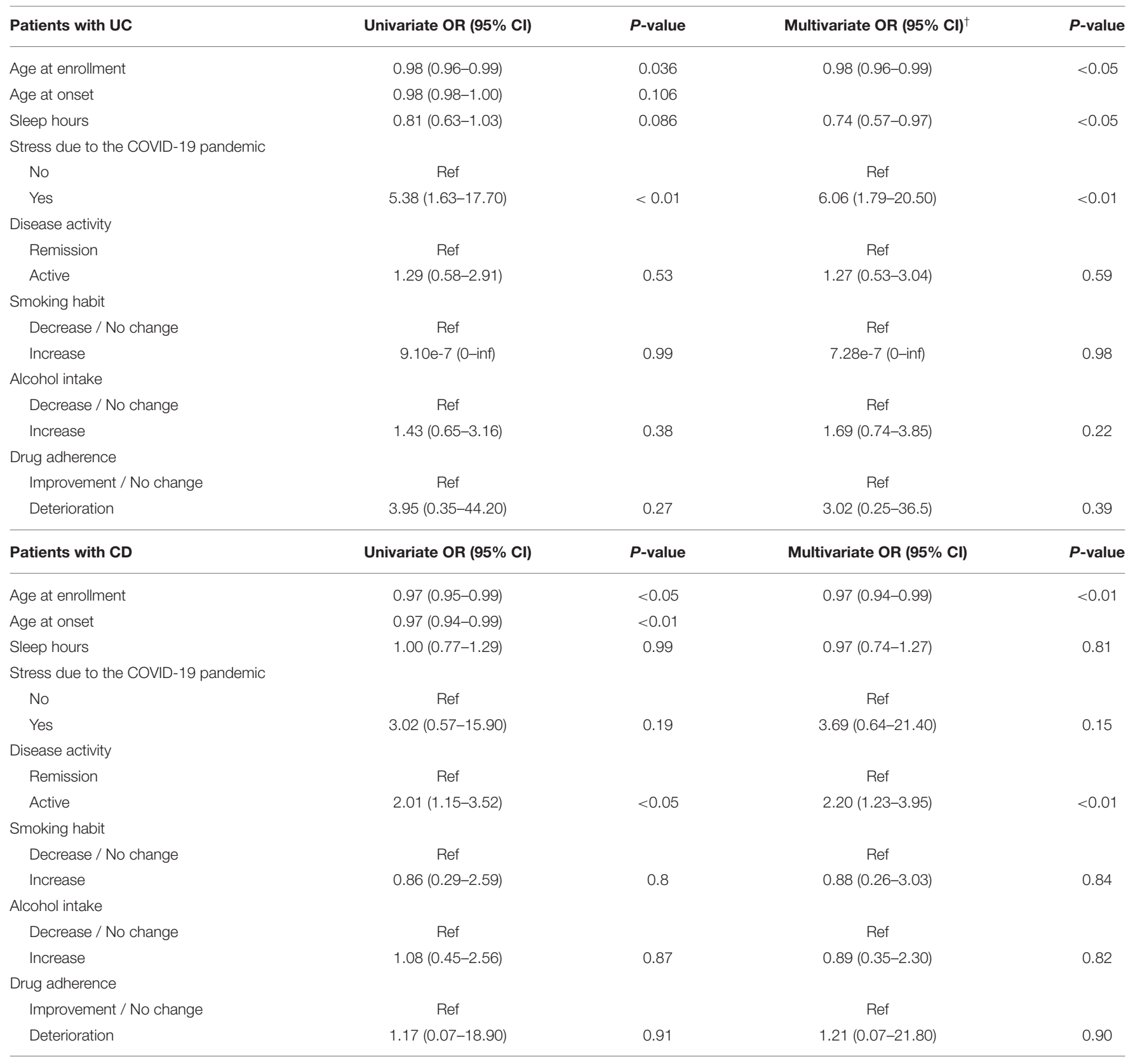

Age at enrollment, age at onset and sleep hours were considered as continuous variables. ${ }^{\dagger}$ Adjusted for factors, including age at enrollment, sex, short sleep, stress due to the COVID-19 pandemic, increased smoking, increased alcohol intake, drug adherence deterioration, and active disease. COVID-19, coronavirus disease; PRO2, patient-reported outcome 2; UC, ulcerative colitis; $C D$, Crohn's disease; $C l$, confidence interval; $O R$, odds ratio.

almost all patients with IBD visit the clinic regularly even if they have no symptoms. In addition, it is difficult to include patients with IBD without clinical visits. Although our hospital is a tertiary medical institution, it also provides regular follow-up for patients with remission or mild IBD. Therefore, this may only moderately limit the generalizability of the findings.

Furthermore, as the questionnaire was completed based on memory recall, a response bias could have influenced the answers of the study participants. This may be owing to fatigue from answering many questions or difficulty in remembering gastrointestinal symptoms or lifestyles before the state of emergency; thus, 60 of 511 participants (11.7\%) could not complete the questionnaire. As this was a retrospective study, a possibility of reverse causality may have occurred. It is possible that patients were having more symptoms from their disease due to exacerbation, which in turn led to poor sleep and increasing stress. Therefore, further large prospective studies are needed to confirm the impact of a lockdown on patients with IBD.

This study is the first to provide data on the association between IBD activity and lifestyle changes/psychological stress 
due to the state of emergency during the COVID-19 pandemic. Our finding suggests that improving the quality of sleep and preventing psychological stress may be significant factors in improving IBD management during a pandemic, especially among young patients.

\section{DATA AVAILABILITY STATEMENT}

The raw data supporting the conclusions of this article will be made available by the authors, without undue reservation.

\section{ETHICS STATEMENT}

The studies involving human participants were reviewed and approved by the Ethics Committee of the Osaka City University Graduate School of Medicine. Written informed consent from

\section{REFERENCES}

1. Wang C, Horby PW, Hayden FG, Gao GF. A novel coronavirus outbreak of global health concern. Lancet. (2020) 395:4703. doi: 10.1016/S0140-6736(20)30185-9

2. Looi MK. Covid-19: Japan ends state of emergency but warns of "new normal". BMJ. (2020) 369:m2100. doi: 10.1136/bmj.m2100

3. Sugaya N, Yamamoto T, Suzuki N, Uchiumi C. A real-time survey on the psychological impact of mild lockdown for COVID-19 in the Japanese population. Sci Data. (2020) 7:372. doi: 10.1038/s41597-020-00714-9

4. Pierce M, Hope H, Ford T, Hatch S, Hotopf M, John A, et al. Mental health before and during the COVID-19 pandemic: a longitudinal probability sample survey of the UK population. Lancet Psychiatry. (2020) 7:88392. doi: 10.1016/S2215-0366(20)30308-4

5. Zhou X, Snoswell CL, Harding LE, Bambling M, Edirippulige S, Bai X, et al. The role of telehealth in reducing the mental health burden from COVID-19. Telemed J E Health. (2020) 26:377-9. doi: 10.1089/tmj.2020.0068

6. Losada-Baltar A, Jiménez-Gonzalo L, Gallego-Alberto L, Pedroso-Chaparro MDS, Fernandes-Pires J, Márquez-González M. 'We are staying at home'. Association of self-perceptions of aging, personal and family resources and loneliness with psychological distress during the lock-down period of COVID-19. J Gerontol B Psychol Sci Soc Sci. (2021) 76:e106. doi: 10.1093/geronb/gbaa048

7. Mazza C, Ricci E, Biondi S, Colasanti M, Ferracuti S, Napoli C, et al. A nationwide survey of psychological distress among Italian people during the COVID-19 pandemic: immediate psychological responses and associated factors. Int J Environ Res Public Health. (2020) 17:3165. doi: 10.3390/ijerph17093165

8. Tang W, Hu T, Hu B, Jin C, Wang G, Xie C, et al. Prevalence and correlates of PTSD and depressive symptoms one month after the outbreak of the COVID19 epidemic in a sample of home-quarantined Chinese university students. $J$ Affect Disord. (2020) 274:1-7. doi: 10.1016/j.jad.2020.05.009

9. Ananthakrishnan AN. Epidemiology and risk factors for IBD. Nat Rev Gastroenterol Hepatol. (2015) 12:205-17. doi: 10.1038/nrgastro.2015.34

10. Ranjbaran Z, Keefer L, Farhadi A, Stepanski E, Sedghi S, Keshavarzian A. Impact of sleep disturbances in inflammatory bowel disease. J Gastroenterol Hepatol. (2007) 22:1748-53. doi: 10.1111/j.1440-1746.2006.04820.x

11. Ali T, Madhoun MF, Orr WC, Rubin DT. Assessment of the relationship between quality of sleep and disease activity in inflammatory bowel disease patients. Inflamm Bowel Dis. (2013) 19:2440-3. doi: 10.1097/MIB.0b013e3182a0ea54

12. Saadoune N, Peyrin-Biroulet L, Baumann C, Bigard MA, Wirth N, Martinet $\mathrm{Y}$, et al. Beliefs and behaviour about smoking among inflammatory bowel disease patients. Eur J Gastroenterol Hepatol. (2015) 27:797803. doi: 10.1097/MEG.0000000000000371 the participants' legal guardian/next of kin was not required to participate in this study in accordance with the national legislation and the institutional requirements.

\section{AUTHOR CONTRIBUTIONS}

SH conceived the study and supervised the overall study. $\mathrm{YNi}$ and $\mathrm{SH}$ wrote the manuscript. Data collection and analysis were performed by $\mathrm{YNi}, \mathrm{SH}, \mathrm{KF}, \mathrm{SI}, \mathrm{NK}$, and $\mathrm{SO}$. All authors contributed to the article and approved the submitted version.

\section{ACKNOWLEDGMENTS}

We thank all the patients that completed the survey.

13. Uemura R, Fujiwara $Y$, Iwakura N, Shiba $M$, Watanabe K, Kamata $\mathrm{N}$, et al. Sleep disturbances in Japanese patients with inflammatory bowel disease and their impact on disease flare. Springerplus. (2016) 5:1792. doi: 10.1186/s40064-016-3408-6

14. Bewtra M, Brensinger CM, Tomov VT, Hoang TB, Sokach CE, Siegel CA, et al. An optimized patient-reported ulcerative colitis disease activity measure derived from the Mayo score and the simple clinical colitis activity index. Inflamm Bowel Dis. (2014) 20:1070-8. doi: 10.1097/MIB.0000000000000053

15. Lewis JD, Chuai S, Nessel L, Lichtenstein GR, Aberra FN, Ellenberg $\mathrm{JH}$. Use of the noninvasive components of the Mayo score to assess clinical response in ulcerative colitis. Inflamm Bowel Dis. (2008) 14:16606. doi: 10.1002/ibd.20520

16. Khanna R, Zou G, D'Haens G, Feagan BG, Sandborn WJ, Vandervoort MK, et al. A retrospective analysis: the development of patient reported outcome measures for the assessment of Crohn's disease activity. Aliment Pharmacol Ther. (2015) 41:77-86. doi: 10.1111/apt.13001

17. Fries W, Viola A, Manetti N, Frankovic I, Pugliese D, Monterubbianesi R, et al. Disease patterns in late-onset ulcerative colitis: results from the IG-IBD “AGED study". Dig Liver Dis. (2017) 49:17-23. doi: 10.1016/j.dld.2016.09.006

18. Ananthakrishnan AN, Shi HY, Tang W, Law CC, Sung JJ, Chan FK, et al. Systematic review and meta-analysis: phenotype and clinical outcomes of older-onset inflammatory bowel disease. J Crohns Colitis. (2016) 10:122436. doi: 10.1093/ecco-jcc/jjw054

19. Nguyen GC, Bernstein CN, Benchimol EI. Risk of surgery and mortality in elderly-onset inflammatory bowel disease: a population-based cohort study. Inflamm Bowel Dis. (2017) 23:218-23. doi: 10.1097/MIB.0000000000 000993

20. Lin WC, Tung CC, Lin HH, Lin CC, Chang CW, Yen HH, et al. Elderly adults with late-onset ulcerative colitis tend to have atypical, milder initial clinical presentations but higher surgical rates and mortality: a Taiwan Society of Inflammatory Bowel Disease study. J Am Geriatr Soc. (2016) 64:e957. doi: $10.1111 /$ jgs.14427

21. Jeuring SF, van den Heuvel TR, Zeegers MP, Hameeteman WH, Romberg-Camps MJ, Oostenbrug LE, et al. Epidemiology and long-term outcome of inflammatory bowel disease diagnosed at elderly age-an increasing distinct entity? Inflamm Bowel Dis. (2016) 22:1425-34. doi: 10.1097/MIB.0000000000000738

22. Charpentier C, Salleron J, Savoye G, Fumery M, Merle V, Laberenne JE, et al. Natural history of elderly-onset inflammatory bowel disease: a populationbased cohort study. Gut. (2014) 63:423-32. doi: 10.1136/gutjnl-2012-303864

23. Graff LA, Vincent N, Walker JR, Clara I, Carr R, Ediger J, et al. A populationbased study of fatigue and sleep difficulties in inflammatory bowel disease. Inflamm Bowel Dis. (2011) 17:1882-9. doi: 10.1002/ibd.21580

24. Ananthakrishnan AN, Khalili H, Konijeti GG, Higuchi LM, de Silva P, Fuchs CS, et al. Sleep duration affects risk for ulcerative colitis: a 
prospective cohort study. Clin Gastroenterol Hepatol. (2014) 12:187986. doi: 10.1016/j.cgh.2014.04.021

25. Ananthakrishnan AN, Long MD, Martin CF, Sandler RS, Kappelman MD. Sleep disturbance and risk of active disease in patients with Crohn's disease and ulcerative colitis. Clin Gastroenterol Hepatol. (2013) 11:96571. doi: 10.1016/j.cgh.2013.01.021

26. Kurina LM, Goldacre MJ, Yeates D, Gill LE. Depression and anxiety in people with inflammatory bowel disease. J Epidemiol Commun Health. (2001) 55:716-20. doi: 10.1136/jech.55.10.716

27. Mancina RM, Pagnotta R, Pagliuso C, Albi V, Bruno D, Garieri P, et al. Gastrointestinal symptoms of and psychosocial changes in inflammatory bowel disease: a nursing-led cross-sectional study of patients in clinical remission. Medicina. (2020) 56:45. doi: 10.3390/medicina560 10045

28. Lewis K, Marrie RA, Bernstein CN, Graff LA, Patten SB, Sareen J, et al. The prevalence and risk factors of undiagnosed depression and anxiety disorders among patients with inflammatory bowel disease. Inflamm Bowel Dis. (2019) 25:1674-80. doi: 10.1093/ibd/izz045

29. Monteleone G, Ardizzone S. Are patients with inflammatory bowel disease at increased risk for Covid-19 infection? J Crohns Colitis. (2020) 14:13346. doi: 10.1093/ecco-jcc/jjaa061

30. Scribano ML. Why do immunosuppressed patients with inflammatory bowel disease not seem to be at a higher risk of COVID19? Dig Dis Sci. (2020) 66:2855-64. doi: 10.1007/s10620-02006624-5

31. Brenner EJ, Ungaro RC, Gearry RB, Kaplan GG, Kissous-Hunt M, Lewis JD, et al. Corticosteroids, but not TNF antagonists, are associated with adverse COVID-19 outcomes in patients with inflammatory bowel diseases: results from an international registry. Gastroenterology. (2020) 159:48191.e3. doi: $10.1053 /$ j.gastro.2020.05.032
32. Wang B, Li R, Lu Z, Huang $\mathrm{Y}$. Does comorbidity increase the risk of patients with COVID-19: evidence from meta-analysis. Aging. (2020) 12:6049-57. doi: 10.18632/aging.103000

33. Li X, Xu S, Yu M, Wang K, Tao Y, Zhou Y, et al. Risk factors for severity and mortality in adult COVID-19 inpatients in Wuhan. J Allergy Clin Immunol. (2020) 146:110-8. doi: 10.1016/j.jaci.2020.04.006

34. Magro F, Rahier JF, Abreu C, MacMahon E, Hart A, van der Woude CJ, et al. Inflammatory bowel disease management during the COVID-19 outbreak: the ten do's and don'ts from the ECCO-COVID taskforce. J Crohns Colitis. (2020) 14 (Suppl. 3):S798-806. doi: 10.1093/ecco-jcc/jjaa160

Conflict of Interest: The authors declare that the research was conducted in the absence of any commercial or financial relationships that could be construed as a potential conflict of interest.

Publisher's Note: All claims expressed in this article are solely those of the authors and do not necessarily represent those of their affiliated organizations, or those of the publisher, the editors and the reviewers. Any product that may be evaluated in this article, or claim that may be made by its manufacturer, is not guaranteed or endorsed by the publisher.

Copyright () 2021 Nishida, Hosomi, Fujimoto, Nakata, Sugita, Itani, Nadatani, Fukunaga, Otani, Tanaka, Nagami, Taira, Kamata, Watanabe, Ohfuji and Fujiwara. This is an open-access article distributed under the terms of the Creative Commons Attribution License (CC BY). The use, distribution or reproduction in other forums is permitted, provided the original author(s) and the copyright owner(s) are credited and that the original publication in this journal is cited, in accordance with accepted academic practice. No use, distribution or reproduction is permitted which does not comply with these terms. 